\title{
Performance Evaluation of Land Mobile Satellite System over Lutz Fading Channel for BPSK Modulation Scheme
}

\author{
Suvarna Subhedar \\ AKGEC, Ghaziabad
}

\author{
Pradeep Kumar Chopra \\ AKGEC, Ghaziabad
}

\begin{abstract}
In this paper, different statistical models of land mobile satellite channel are studied with emphasis on Lutz Model. The reason for selecting Lutz Model is that this model shows best distribution in the entire environment (open area, semi shadowed and shadowed region). The Lutz Channel Model is simulated in Matlab using random number generator technique and is validated. The channel model is used to evaluate the performance of satellite system in terms of Bit error rate using BPSK modulation scheme and is compared with AWGN, Rician and Rayleigh channel.
\end{abstract}

\section{General Terms}

Land mobile satellite channel modeling

\section{Keywords}

Bit error rate (BER), land mobile satellite system, multipath fading, propagation channel

\section{INTRODUCTION}

Now-a-days researches are going on for efficient utilization of artificial satellite in telecommunication fields. This is because satellite provides large coverage area and also high data rate irrespective of the user's geographical location [1]. In a communication Engineer's point of view, it needs to be known what are the relative signal loss, frequency drifts, timing slips and phase variations of a signal when transmitted through a channel. Land mobile satellite channels mainly vary with the geographical structures and sites, weather conditions and mobility of the transmitter with respect to the receiver. Researchers model such channels statistically and characterize them depending on how severe the fading effects are on the signal. In a design Engineer's perspective, it is desirable to test their transmitter and receiver designs to determine the performance prior to building prototypes or manufacturing [2].

In free space, the propagation loss between a transmitter and receiver is readily predicted. In most applications however, propagation is impaired by proximity to the earth, objects blocking the LoS and/or atmospheric effects. Because of these impairments, the fundamental characteristics of RF propagation generally vary with the frequency of the electromagnetic wave being propagated. The frequency spectrum is grouped into bands, which are designated by abbreviations such as HF, VHF, and so on. Letter designations of the bands are also used, although the definitions can vary.

Propagation of electromagnetic waves may occur by ground wave, tropospheric wave, or sky wave. Most contemporary communication systems use either direct LoS or indirect propagation, where the signals are strong enough to enable communication by reflection, diffraction, or scattering as shown in Figure 1. Ionospheric and tropospheric propagation are rarely used, and the effects tend to be treated as nuisances rather than a desired means of propagation.

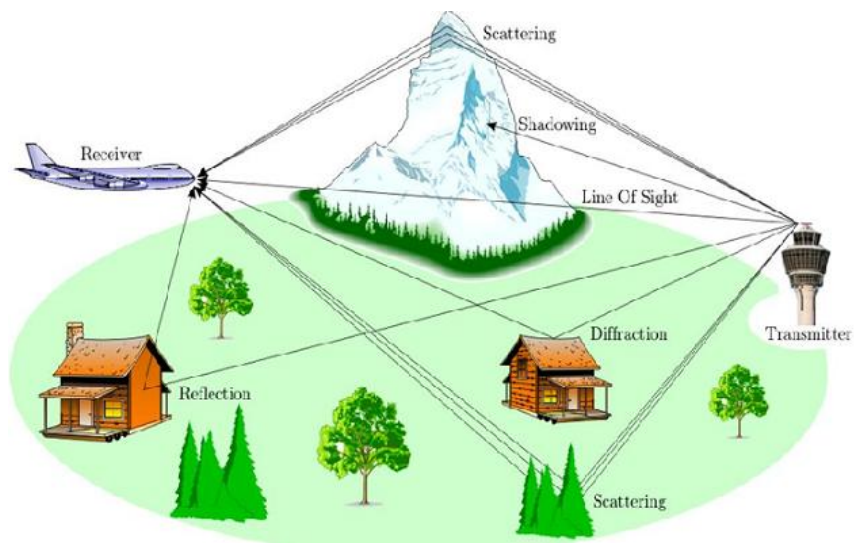

Fig.1: Mutlipath Propagation

When there is no obstacle in between transmitter and receiver, i.e. LoS signal is only present then the channel is known as AWGN (Adaptive White Gaussian Noise) channel. If the signals is obstructed by some obstacle but at the receiver end both LoS and obstructed signal received, then fading channel is treated as Rician channel. And the case when there is no LoS component means signal reaches to receiver through reflection, refraction and scattering. The strength of obstructed signal is more than threshold then the fading channel is treated as Rayleigh Channel. The four main distributions used by different literatures in different proportion for designing different channel are:

\section{Lognormal Distribution}

Rician Distribution

Rayleigh Distribution

Gaussian Distribution

Figure 2 (a) and 2 (b) shows the PDF (probability distribution function) for lognormal distribution and remaining three distributions, respectively. 


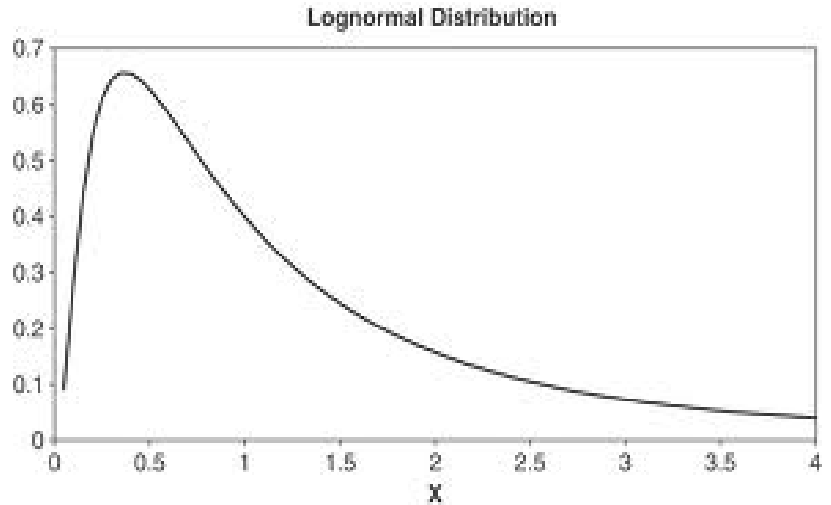

(a)

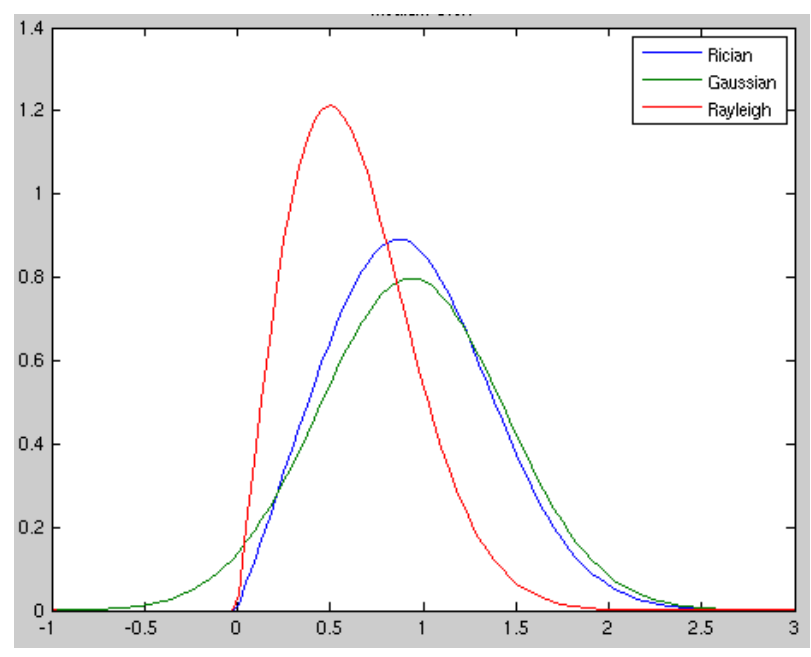

(b)

Fig. 2 (a), (b): PDF for different distributions

This paper is organized as follows. In Section 2, the general Channel models based on the propagation scattering theory are derived. In Section 3, the probability density functions (pdf's) of the received signal envelope and the received signal power are given for the Lutz Channel. In Section 4, the software simulator used to measure the performance of LMSS system is explained. In Section 5, numerical results for analytical data fitting for the multistate model (Lutz model) in land-mobile and mobile satellite communications are presented. The channel is then used to calculate BER and the result is compared with different channel model. Section 6 provides the brief conclusion.

\section{CHANNEL MODELING}

The narrow-band models reported in the literature can be classified into three main categories: empirical, statistical and analytical [3].The statistical modeling of LMS communication channel has seen a growing interest in recent years. Ossanna [4] was the first to attempt to give a reasonable statistical characterization on the received mobile-radio signals using a set of interfering waves for land mobile communication environment. After introducing the concept of large-scale fading and small-scale fading, Suzuki [5] gave the Rayleigh lognormal model that was well tested in built-up areas and showed that this model was more appropriate for fitting the experimental data. Loo [6] proposed a model suitable for rural environments, in which it assumed that the received signal was affected by frequency-non selective Rice fading with lognormal shadowing on the direct component, the resulting model gave a generalization of the Suzuki's model. Along a similar line, Corazza and Vatalaro [7] presented another Ricelognormal distribution, in which the shadowing affects both direct and diffuse components. All models discussed above are suitable for cases when the receiving terminals are fixed or mobile in a uniform environment - we call the model suitable for this environment the single-state model. When the terminals travel in a large area of a non uniform environment, the received signals may change abruptly at different power levels, therefore, the received signals are more appropriately characterized by the what-so-called multistate model. Lutz et al. [8] introduced a two-state model, in which it was assumed that the received signals can experience two states: the "good state," corresponding to the case when the signals only undergo shadowing, and the "bad state," corresponding to the case when the signals experience strong component attenuation and weak signal fading.

Alireza Mehrnia [9], studied several major mobile satellite propagation channel models and their performances were compared with empirical distributions by means of extensive computer simulations. Table 1 presents the Mean Square Error (MSE) between empirical distributions and the five models for the Open Area, Shadowed Area, and Semi-open, Semi-Shadowed areas. The last column of this Table also presents relative performance of the five models. The major conclusion was that Lutz, Nakagami, Corazza, Loo, and Norton represent the better performance models, in the given order.

Table 1. Least Mean Square Error between Empirical and Theoretical Distributions [9]

\begin{tabular}{|l|l|l|l|l|l|}
\hline Model & $\begin{array}{l}\text { Open } \\
\text { Area }\end{array}$ & $\begin{array}{l}\text { Shadowe } \\
\text { d Area }\end{array}$ & $\begin{array}{l}\text { Semi- } \\
\text { Open } \\
\text { Semi- } \\
\text { Shadow } \\
\text { ed }\end{array}$ & $\begin{array}{l}\text { Overall } \\
\text { Perfor } \\
\text { mance }\end{array}$ & $\begin{array}{l}\text { Relative } \\
\text { MSE }\end{array}$ \\
\hline Norton & 0.8737 & 1.3680 & 1.4593 & 1.2340 & 1.8 \\
\hline Loo & 0.8107 & 1.2554 & 1.4365 & 1.1680 & 1.7 \\
\hline Corazza & 0.8177 & 0.9373 & 0.9316 & 0.8960 & 1.3 \\
\hline $\begin{array}{l}\text { Nakaga } \\
\text { mi }\end{array}$ & 0.5310 & 1.0890 & 0.8622 & 0.8270 & 1.2 \\
\hline Lutz & 0.4545 & 0.7823 & 0.8290 & 0.6890 & 1.0 \\
\hline
\end{tabular}

So, in this paper Lutz channel is used for evaluating the performance of LMS system during shadowing.

\section{LUTZ CHANNEL}

Different from the fixed service satellite system, LMSS experiences multipath fading and shadowing by terrain obstacles. Shadowing is the dominant fading mechanism for a LMSC [10]. Thus, the received signal at the terrestrial mobile terminal is classified into two types, the unshadowed signal and the shadowed signal, according to the obstacles in the line-of-sight path between a satellite and a mobile terminal.Thus, Lutz model is two state model in which Good State is represented by Rician Distribution (unshadowed signal) having an LoS component present. In bad state, LoS 
path is blocked through obstacles (like buildings, trees) and the signal is received through multipath component which is $\log$ normally distributed. In this case received signal is combination of Rayleigh and lognormal distribution i.e. Suzuki distribution (Shadowed signal).

Generally, the propagation path for a mobile terminal includes both unshadowed and shadowed propagation conditions [11]. The cumulative distribution is the sum of the shadowed and unshadowed distribution, weighted by the fraction of shadowing $S$.

$$
\mathrm{G}(\mathrm{F})=S * \mathrm{G}_{\text {suzuki }}+(1-\mathrm{S}) * \mathrm{G}_{\text {rice }}
$$

In this paper, the cumulative fade distribution, CFD $(\mathrm{F})$ is used as a primary statistics in evaluating the satellite propagation link.

$$
C F D(F)=1-G(F)
$$

where $F=-R$ is fade level.

\section{SOFTWARE SIMULATOR}

Channel simulators have been used extensively as an effective means for the evaluation of satellite link performance and also helpful in reduction of experimental cost.

The concept of designing a Channel Simulator is taken from [11]. In the simulator, each data set, such as Rayleigh, Rician, Lognormal and the shadowed data set is generated by the RNG (Random Number Generator) in MATLAB. The fraction of shadowing $S$, which is used as input parameter for the simulator. The outputs of the simulator are plots of data samples, PDF and CFD. The block diagram of the propagation channel simulator is depicted in Figure 3.

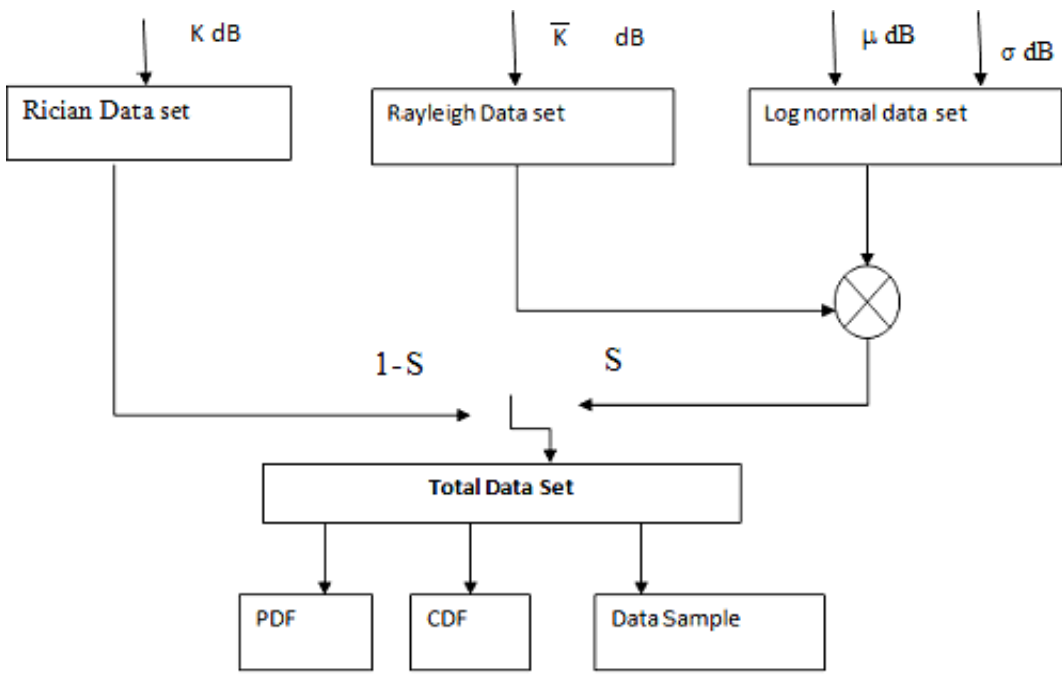

Fig. 3: Block Diagram of Channel Simulator

\section{RESULTS AND DISCUSSION}

The simulations of the fading channels are carried out in MATLAB software. MATLAB codes are written for the implementation of the models and are run to generate results in the form of graphs for visualization.

Figure 4 shows the plots of the $C F D$ predicted by the analytical model [12] and the simulated data set generated by the simulator proposed in this paper, (a), (b) and (c) corresponds to the UHF-band light shadowing, L-band middle shadowing and Ka-band heavy shadowing, respectively. In spite of some little deviation comparing with the analytical model statistics at large fade levels, the simulation results of the simulator show excellent agreements with the analytical model under the three frequency bands shadowing conditions. The parameters used for Figure. 3 are in Table 2.

Based on the parameters of Table 2, the envelope CFDs for Lutz's model and analytical model is plotted. A close agreement between the two models is depicted in Figure 3 over a wide range of signal levels, for several different sets of data collected at different places, frequency bands, and elevation angles, is excellent. This strongly supports the application of the proposed model for LMS channels.
The system used for evaluation of the error performance of BPSK over the simulated Lutz channel is shown in figure 5. In this system only the magnitude of the fading process is considered, which means that the probability of error is the probability of error due to amplitude fading.

The focus is on an error probability of $10^{-3}$ as this is the target BER in speech applications. For the degree of shadowing the SNR penalties, which are substantial when compared to AWGN case, are shown in Table 3.

Table 2. Parameters for Figure 3 [3]

\begin{tabular}{|c|c|c|c|c|c|}
\hline Conditions & $\mathbf{S} \%$ & $\mathbf{K ~ d B}$ & $\mathbf{K}^{-} \mathbf{d B}$ & $\boldsymbol{\mu ~ d B}$ & $\boldsymbol{\sigma} \mathbf{d B}$ \\
\hline UHF band & 6 & 13 & 4.4 & -4 & 4.9 \\
\hline L band & 50 & 5 & 3 & -0.5 & 5 \\
\hline Ka band & 92.5 & 21.5 & 9.9 & -4.8 & 1.7 \\
\hline
\end{tabular}




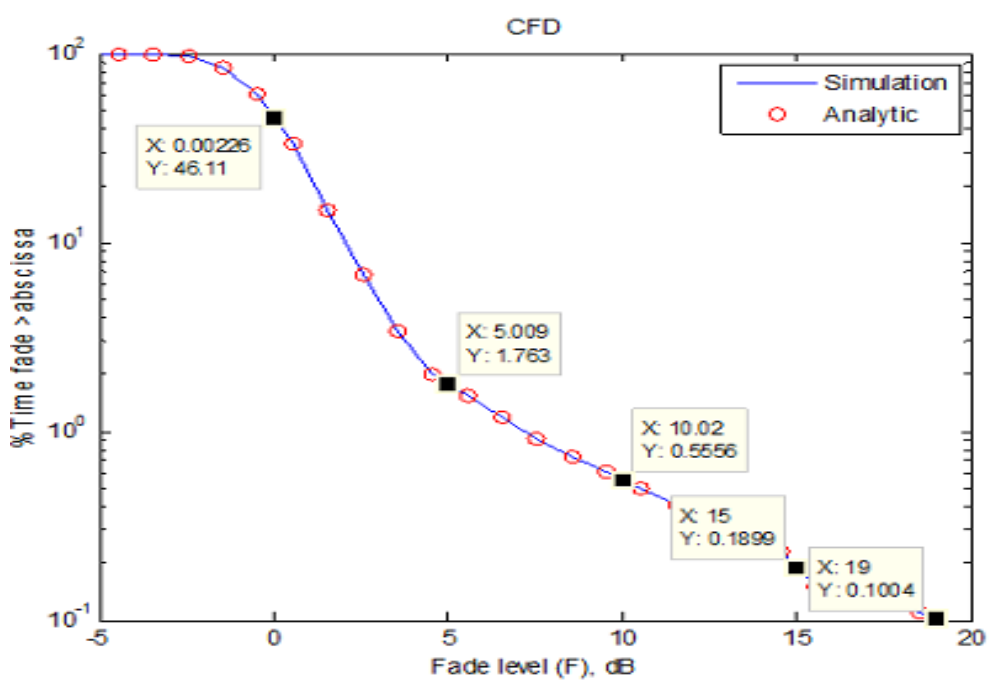

(a) UHF Light Shadowing

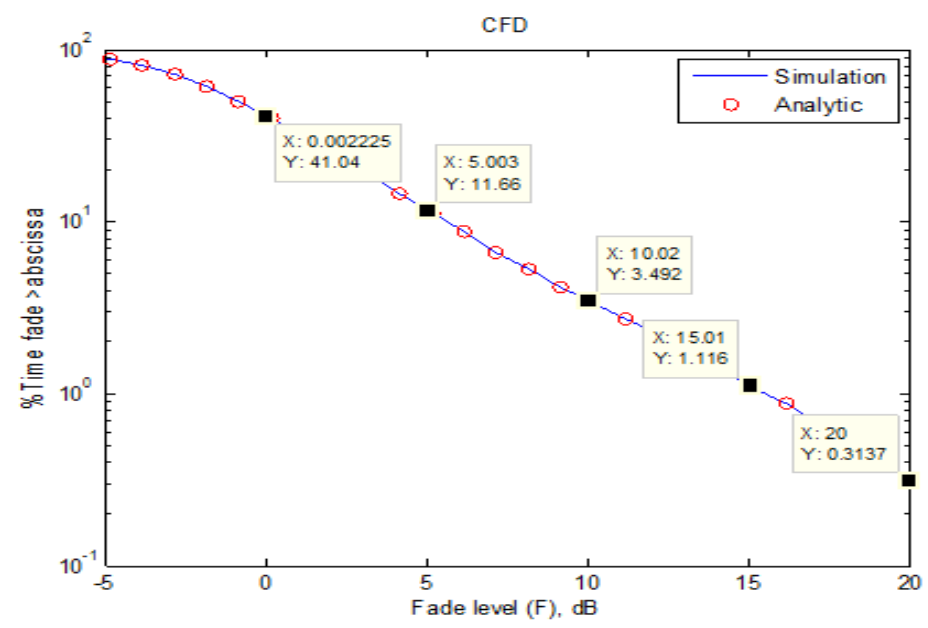

(b) L band Moderate Shadowing

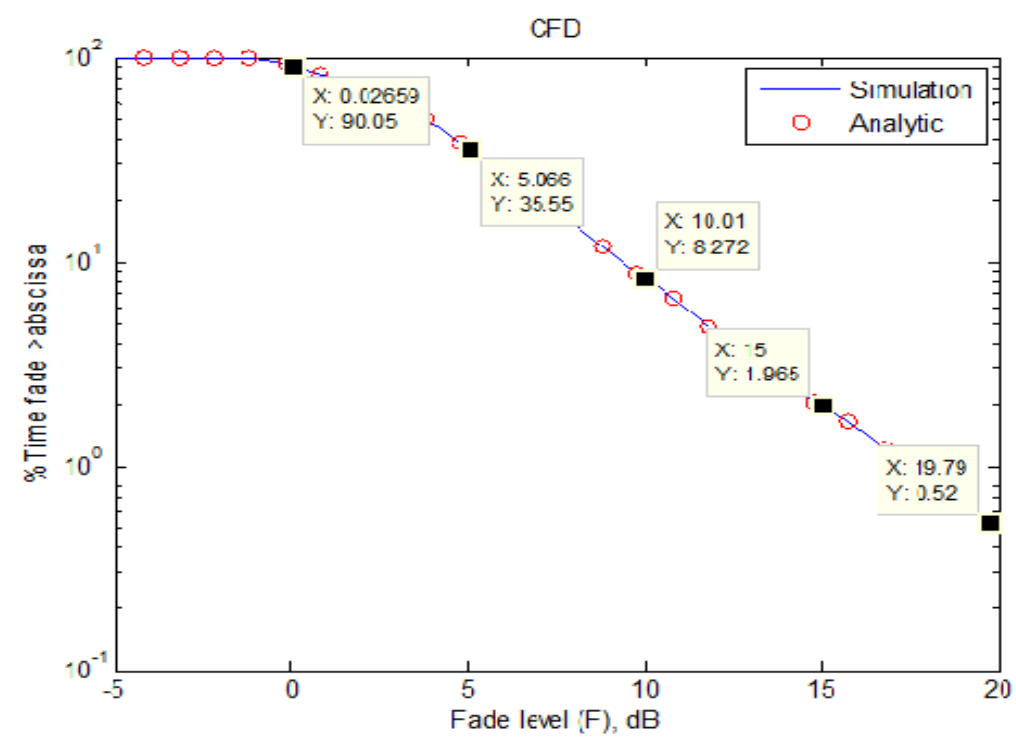

(c) Ka-band Heavy Shadowing

Fig. 4: Comparisons of CFD between the simulator and the analytical model 
Table 3. SNR degradation due to fading

\begin{tabular}{|c|c|c|}
\hline Degree of Shadowing & \multicolumn{2}{|c|}{ SNR penalties (dB) } \\
\cline { 2 - 3 } & $\mathrm{P}_{\mathrm{e}}=10^{-3}$ & $\mathrm{P}_{\mathrm{e}}=10^{-4}$ \\
\hline Light Shadowing & 7 & 15 \\
\hline Middle Shadowing & 13 & 23 \\
\hline Heavy Shadowing & 15 & 24 \\
\hline
\end{tabular}

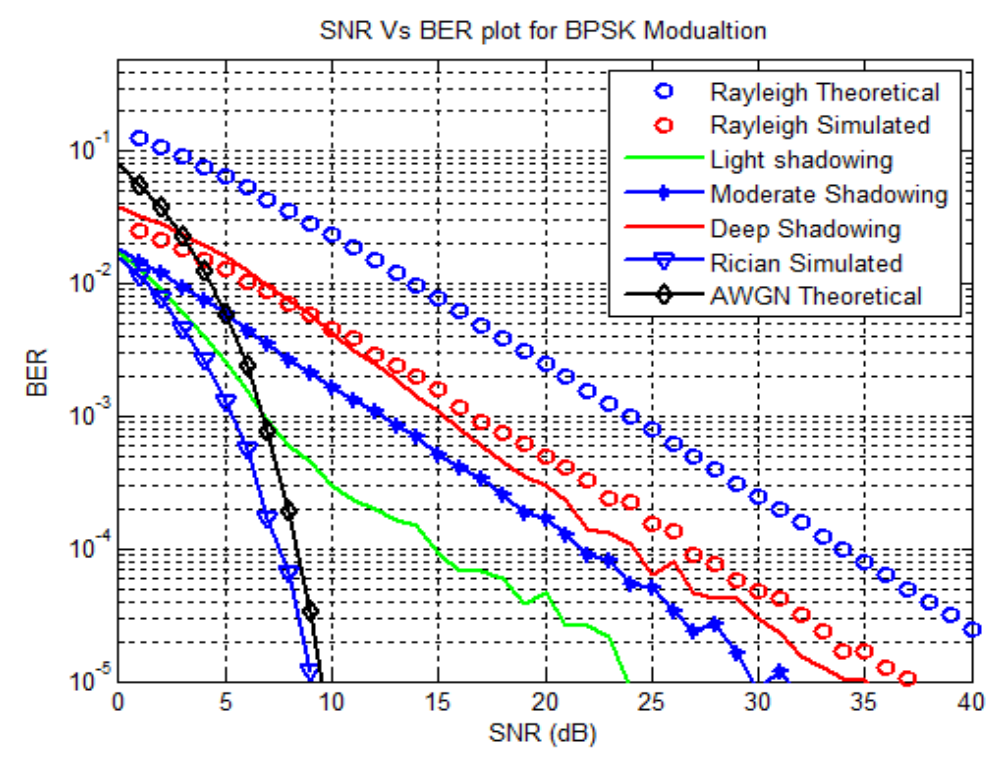

Fig. 5: BER comparison of all the Fading Channels

\section{CONCLUSION}

This paper investigates a two-state simulation model for a frequency-selective land mobile satellite communication (LMSC) channel. Aside from ionospheric effects, the propagation channels for LMSC systems are characterized by wideband effects due to multipath fading which makes the channels time-variant and exhibit frequency-selective distortion. Hence, an adequate knowledge and modelling of the propagation channel is necessary for the design and performance evaluation of the LMSC systems. A two-state simulation model for a frequency-selective LMSC channel, which is a combination of Rayleigh and Rician fading processes, is developed. The propagation characteristics of the proposed LMSC channel model are presented, and comparisons are made with the Rayleigh, Rician and AWGN fading channels using bit error rate (BER) as the figure of merit. The simulation results show that the degree of fading experienced by the LMSC link depends on the percentage of shadowing and it is observed from the BER results that the propagation impairment of the LMSC fading channel is relatively lower than that of Rayleigh fading channels but higher than the Rician and AWGN fading channel.

\section{ACKNOWLEDGMENT}

The authors sincerely thank Department of Electronics and Communication Engineering of Ajay Kumar Garg Engineering College, Ghaziabad for providing the opportunity and facilities for performing this research.

\section{REFERENCES}

[1] Zachaeus K. Adeyemo, Olumide O. Ajayi, and Festus K. Ojo, "Simulation Model for a Frequency-Selective Land Mobile Satellite Communication Channel," Innovative Systems Design and Engineering, vol. 3, no.11, pp. 7184, 2012.

[2] S. Kandeepan, and A.D.S. Jayalath, "Narrowband channel simulator based on statistical models implemented on Texas Instruments c6713 DSP and National Instruments pcie6259 hardware," IEEE Singapore International Conference on Communication systems, (ICCS 2006), pp. 1-6, 2006.

[3] M. Karaliopoulos, and F. Pavlidou, "Modelling the land mobile satellite channel: a review," Electronics Communication Engineering Journal, vol. 11, no. 5, pp. 235 -248, 1999. 
[4] J. F. Ossanna, "A model for mobile radio fading due to building reflections: Theoretical and experimental fading waveform power spectra," B.S.T.J., vol. 43, no. 6, pp. 29352971, Nov. 1964.

[5] H. Suzuki, "A statistical model for urban radio propagation," IEEE Trans. Commun., vol. COM-25, no. 7, pp. 673-680, 1977.

[6] C. Loo, "A statistical model for a land mobile satellite link," IEEE Trans. Veh. Technol., vol. VT-34, no. 3, pp. 122-127, 1985.

[7] G. E. Corazza, and F. Vatalaro, "A statistical model for land mobile satellite channels and its application to nongeostationary orbit system," IEEE Trans. Veh. Technol., vol. 43, no. 3, pp. 738-742, 1994.

[8] E. Lutz, D. Cygan, M. Dippold, F. Dolainsky, and W. Papke, "The land mobile satellite communication channel-Recording, statistics, and channel model," IEEE Trans. Veh. Technol., vol. 40, no. 2, pp. 375-386, 1991.
[9] Alireza Mehrnia Homayoun Hashemi, "Mobile Satellite Propagation Channel Part I - A Comparative Evaluation of Current Models," IEEE Trans. Veh. Technol., pp. 2775-2779, 1999.

[10] R. M. Barts, and W. L. Stutzman, "Modeling and simulation of mobile satellite propagation," IEEE Trans. Antennas and Propagation, vol. 40, pp. 375-382, 1992.

[11] Y. Mingchuan, G. Qing, and W. Zhenyong, "A random number generator based software channel simulator for land mobile satellite channel," Proceedings of the IEEE, pp. 10951098, 2007.

[12] C. Loo, and J. S. Butterworth, "Land mobile satellite channel measurements and modeling," Proceedings of the IEEE, vol. 86, pp. 14421463,1998 